in vivo $36: 30-39(2022)$

doi:10.21873/invivo.12673

Review

\title{
Revision of Roux-en-Y Gastric Bypass for Inadequate Weight Loss or Weight Regain
}

\author{
ANDREAS ALEXANDROU, PANAGIOTIS SAKARELLOS, SPYRIDON DAVAKIS, MICHAIL VAILAS, \\ NIKOLETTA DIMITRIOU, ALEXANDROS PAPALAMPROS, DIMITRIOS SCHIZAS, \\ ALEXANDROS CHARALABOPOULOS and EVANGELOS FELEKOURAS
}

First Department of Surgery, Laiko General Hospital, Medical School of Athens, National and Kapodistrian University of Athens, Athens, Greece

\begin{abstract}
Inadequate weight loss or weight regain after Roux-en-Y gastric bypass (RYGBP) occurs in more than a quarter of patients for various reasons. Available remedying treatment options include endoscopic and surgical techniques for revision of the gastric pouch and the gastrojejunal anastomosis, conversion of standard to distal gastric bypass (DRYGBP) or the conversion of RYGBP to biliopancreatic diversion (BPD) or duodenal switch (DS). There is quite a variability concerning the technical simplicity, safety, and effectiveness of these techniques and the small number of patients in the numerous single-center reports precludes any meaningful comparisons. This review aimed to describe all available methods and present the advantages and disadvantages of each of them, to facilitate, rather than guide, the decision of the average bariatric surgeon who encounters such a patient.
\end{abstract}

Bariatric and metabolic surgery represents currently the most effective therapy of morbid obesity and associated comorbidities, with superior results compared to other ways of treatment such as dietary and life-style modifications or drugs (1). With evolving technologies, increasing experience and standardization of surgical techniques, its safety record is nowadays comparable or even better than any bread-and-

This article is freely accessible online.

Correspondence to: Andreas Alexandrou MD, Ph.D., Associate Professor of Surgery, First Department of Surgery, Laiko General Hospital, Medical School of Athens, National and Kapodistrian University of Athens, 17 Agiou Thoma Str., 11527, Athens, Greece. Tel: +30 6932648746, e-mail: alexandrouandrea@ hotmail.com

Key Words: Obesity, gastric bypass, weight regain, revisional surgery, outcomes, review. butter type of surgery (2). Short and long-term results concerning weight-loss are more than satisfactory (3) with some existing variability for the various types of operations (4). Morbid obesity though is a chronic disease, thus failure of treatment is an inherent phenomenon (5), like in any other chronic disease, i.e., malignancies, immunological disorders etc.

Roux-en-Y gastric bypass (RYGBP) since its first report in the mid-sixties by Mason and Ito (6) and especially after its popularization as a laparoscopic procedure after the works of Wittgrove and Nguyen in the late 90's, has remained the gold-standard for the surgical treatment of morbid obesity for decades (Figure 1). Its technical difficulty has traditionally made RYGBP unattractive both for the not very motivated patients and the average, not devoted bariatric surgeons. Despite this, RYGBP consisted steadily $40-45 \%$ of the total yearly performed operations worldwide, mainly because of its popularity among the majority of bariatric centers in the US (7), before yielding to laparoscopic sleeve gastrectomy as the most popular bariatric operation in 2014 (8).

The main reasons for this supremacy of RYGBP in comparison to other bariatric techniques have always been the constant reduction in the associated peri-operative morbidity and mortality (9), the superb immediate weight loss and amelioration of co-morbidities (10), and last but not least, the sustainability of these results in the long-term along with an excellent quality of life (11).

Identifiable factors for these good results are mainly the combination of mechanisms of action including mechanical restriction of ingested calories due to the small gastric pouch, mild malabsorption due to the by-passing of a reasonable portion of the jejunum, and hormonal changes ensuing from the two latter, like reduction of the production of ghrelin from the excluded gastric fundus, early secretion of peptide YY(PYY) from the distal ileum and changes in the levels of various incretins such as GLP1 (12). 


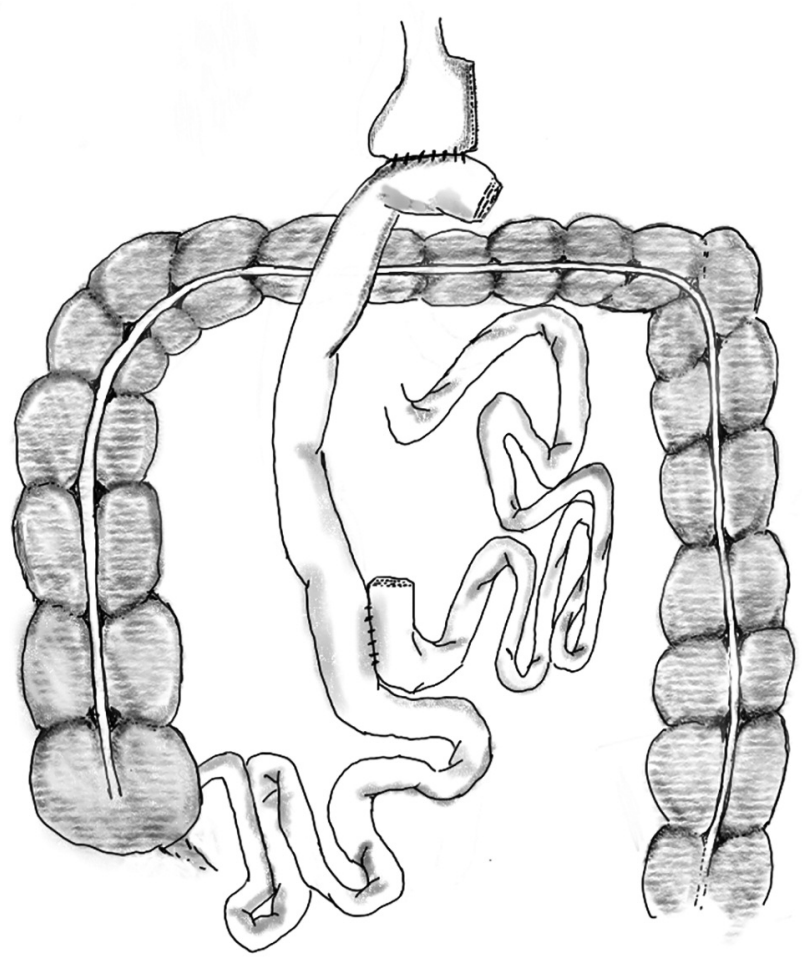

Figure 1. Roux-en-Y gastric bypass.

Despite all these, both primary failure in weight reduction or weight regain after an early success post-RYGBP, although sparse, are well described events and occur in approximately $10-20 \%$ of patients (13). Subjective reasons, like lack of discipline from the patient's part or more objective reasons such as anatomical changes attenuating the mechanism of action of the procedure due to surgical complications such as the occurrence of a gastro-gastric fistula or a gastric pouch enlargement and even hormonal changes, have been hypothesized to be responsible for this failure $(14,15)$, which obviously deteriorates the rate of patient satisfaction from the operation and leads to the recurrence of associated comorbidities that usually accompany obesity.

Last but not least, suboptimal weight loss results occur more often after gastric banding or sleeve gastrectomy (16), thus they represent a better analyzed problem with quite some viable ways of treatment, whereas on the contrary, failure after RYGBP is less common, thus a less extensively studied phenomenon. Also, the complexity of the initial operation makes it even more complicated when the treating physician has to come up with a solution with an acceptable risk-to-benefit ratio and reasonable medium and long-term results concerning weight loss.

The aim of this study was to sum up the existing data and available surgical options for the treatment of patients with unsatisfactory weight loss or weight regain post RYGBP.
Distalization of the Jejuno-Jejunal Anastomosis. Malabsorptive procedures such as biliopancreatic diversion or duodenal switch (BPD/DS) are reportedly associated with a better weight loss and a more beneficial effect on the various co-morbidities of obesity (17). The so called 'distal gastric bypass' (DRYGBP) with a common channel of 100 $\mathrm{cm}$ or even less had been reported as a first line option for super obese patients (18).

Not astonishingly, the conversion of a failed RYGBP to a DRYGBP by reconstructing the jejuno-jejunal anastomosis (JJ) more distally, a configuration that shortens the length of the common channel thus providing increased malabsorption, looks like a very appealing concept and has extensively been reported (19-23) (Figure 2). On the other hand, malabsorptive procedures carry a significant risk for severe nutritional deficiencies often requiring reversion (24). Technically, there are two ways to reposition the JJ of a standard RYGBP, to establish a short common channel thus converting it to a distal gastric bypass. Although both look similar, they are substantially different and inevitably have different post-op results. The JJ can be taken down at the end of the alimentary loop and this loop can then be reanastomosed in a premeasured position at the distal ileum (25). In this way, not only the common channel is shortened but at the same time the bilio-pancreatic loop is elongated, and the additive length of the common and alimentary loop is proportionally decreased.

Alternatively, the existent JJ can be taken down at the biliopancreatic loop's end and the bilio-pancreatic loop be then re-anastomosed more distally $(75 \mathrm{~cm}$ cephalad of the ileocecal valve), thus creating a shorter common channel while both the length of the BP loop and the sum of common and alimentary loops remain unchanged (26). The latter configuration was described by Brolin and as newer usually is referred to as DRYGBP type II, while the former technique had been described earlier by Sugerman with a common channel length of $150 \mathrm{~cm}$, and independently also by Torres as an initial stand-alone operation and had been termed DRYGBP type I.

Both techniques for the revision of standard RYGBP to DRYGBP are technically difficult when performed laparoscopically (27) and for the sake of safety (in order to avoid ischemia of the short intestinal remnants on the side that is taken down), according to some authors, they actually require the total resection of the $\mathrm{JJ}$ and the construction of two new intestinal anastomoses: either one for the reestablishment of the continuity of the alimentary tract and one for the re-implantation of the biliopancreatic loop (type 2) or one for the re-establishment of the continuity of the biliopancreatic limb and one for the re-implantation of the alimentary limb (type 1) (28).

It seems that, with both these techniques the results are excellent and durable even after the first three years post- 


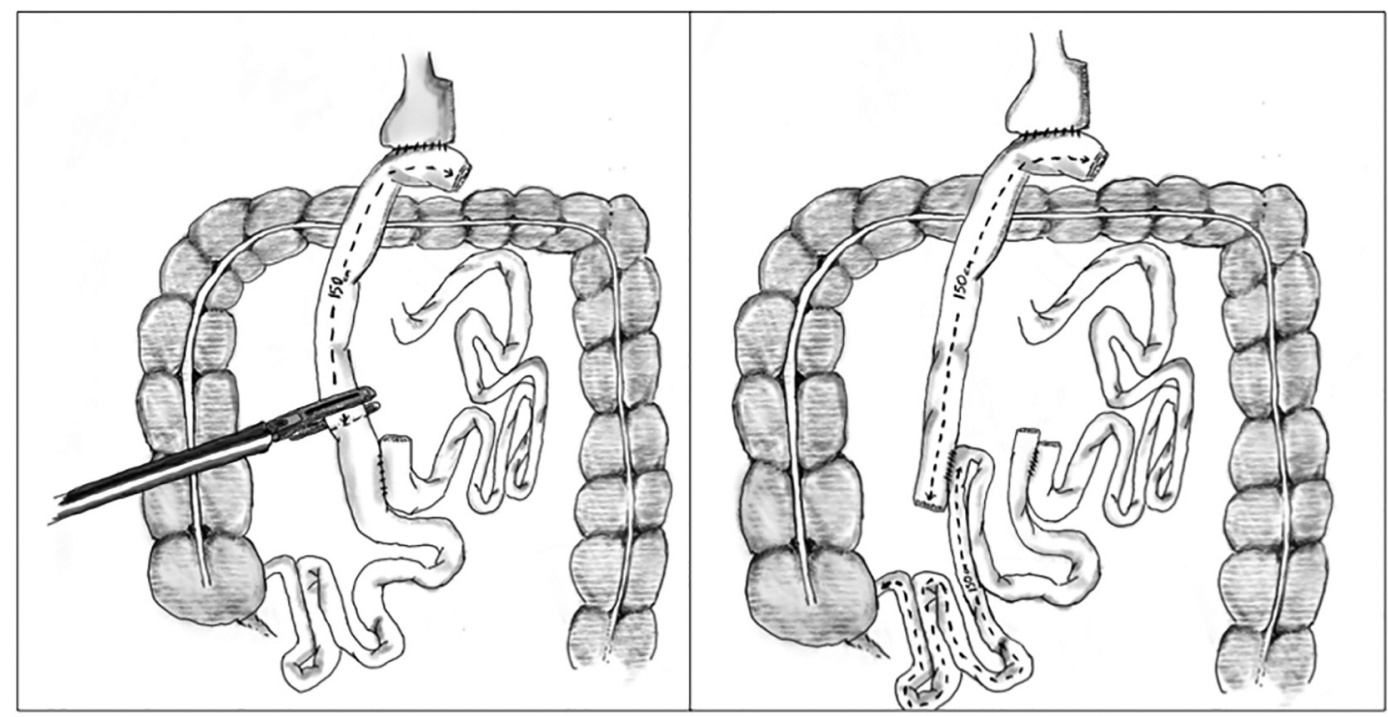

Figure 2. Distalization of the jejuno-jejunal anastomosis.

revision (29). The curve of weight loss is steeper with type I DRYGBP and the quality of life is quite good as long as the length of the common loop is anywhere between 100-150 $\mathrm{cm}$. The rate of excess weight loss (EWL) one-year post-op is reported anywhere between 61 to $90 \%$ and even 5-years post-op it is retained approximately $68-85 \%$ or even $77 \%$ ten years after the revision (21). The downside though is an increased rate of morbidity, which mainly consists of longterm nutritional disorders necessitating parenteral treatment or revision of the distalization. A combined protein and calories malnutrition has been reported to occur between 8 and $31 \%$ and the administration of total parental nutrition (TPN) was necessitated for $14-21 \%$ of patients (29).

For patients with extremely short length of common loop $(50 \mathrm{~cm})$ the outcome was disappointing since all of them required reversal, while even death from liver failure was not unheard of (25). Buchwald et al. have reported their experience with 53 type I revisions of RYGBP to DRYGBP. The rate of excess weight loss was excellent with one third of the total body mass index (BMI) being lost 5 years after the revision. The cost was also not negligible though, since one patient died 2 years post-op due to malnutrition, while $26.4 \%$ of patients had to be revised for the same reason. No patient with a Roux limb length more than $250 \mathrm{~cm}$ had to be revised (28).

More recently, Kralijevic et al. reported their experience with the revision of 24 patients with inadequate weight loss after RYGBP who were converted to distal gastric bypass with the re-implantation of the alimentary loop more distally, and thus the construction of a very long biliopancreatic loop. They constructed a common loop of 90-100 cm with a cumulative length of the alimentary and common loop of 250

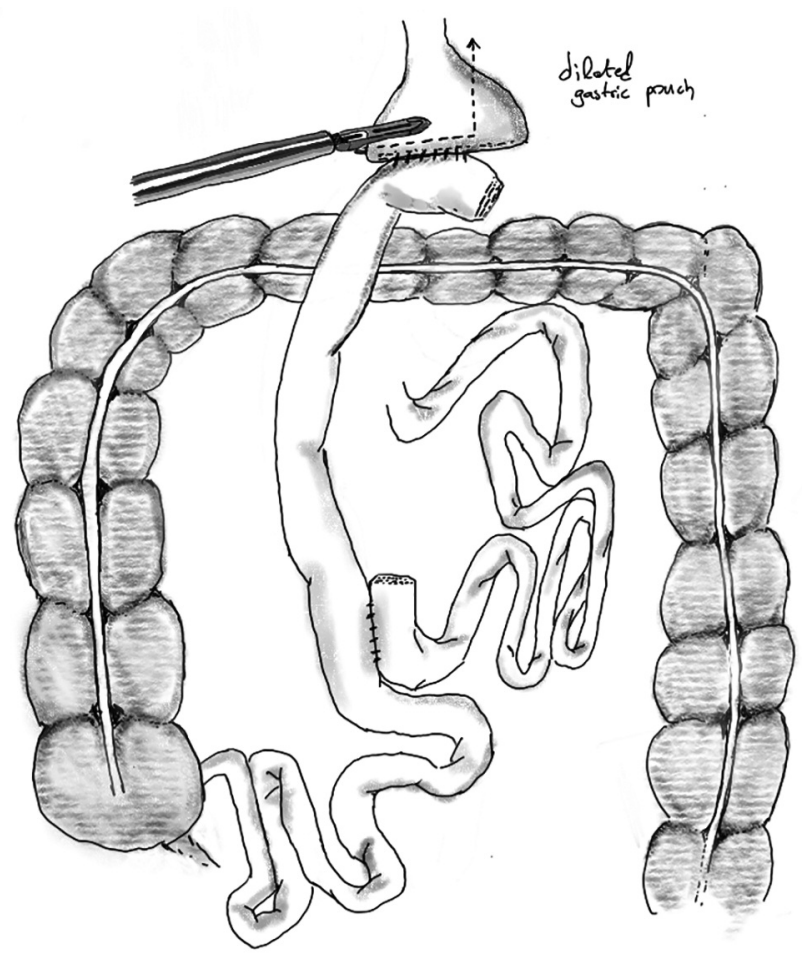

Figure 3. Gastro-jejunostomy anastomosis revision.

$\mathrm{cm}$. The post-operative excess weight loss (EWL) was above $50 \%$ and was retained in the long-term, but $21 \%$ of the patients had to be revised due to malnutrition problems. The authors advocated that after conversion to DRYGBP, the absolute minimum of the length for the sum of alimentary 
and common loop should be no less than $300 \mathrm{~cm}$ to avoid long-term nutritional problems (30).

Pretty similar results were also reported by Shin et al. after revising 21 patients with inadequate weight loss after RYGBP to distal gastric bypass by taking down the JJ at the end of the alimentary limb and re-anastomosing this Roux limb more distally, thus lengthening the BP limb at the cost of the common channel length. The authors concluded that a common channel length of $200 \mathrm{~cm}$ and a cumulative length of $250 \mathrm{~cm}$ for the sum of Roux and common channel provided optimal results, both concerning weight loss and avoiding long-term nutritional deficiencies (31).

On the other hand, with DRYGBP type II the weight loss may be slower or even less profound despite the shorter common channel, but at the same time the absorption of calories and proteins in the elongated alimentary loop retains a better nutritional status both in the short and the long-term. Excess weight loss for the first post-op year was $48 \%$ but malnutrition or need for TPN was kept at a low $7 \%$ and need for revision even less at $6 \%$ (26).

Direct comparison of the two methods to have a definitive result concerning the superiority of either of them is practically impossible due to the low number of cases, which is prohibitive for any meaningful statistical analysis. A more customized approach for every patient (or for every surgeon) seems unavoidable. It seems reasonable to accept that with DRYGBP type I with a very long biliopancreatic limb the absorption of nutrients will be less, thus EWL will be more, along with the chance for malnutrition. On the contrary, with DRYGBP type II with a short common channel but with absorption of nutrients occurring also in the elongated alimentary loop according to the Scopinaro teachings, the rate of EWL will be lower but also with less frequent nutritional deficiencies and the ensuing the need for invasive treatment to ameliorate them.

As of today, the conversion of standard RYGBP to its distal variation is the second-best option to treat unsatisfactory weight loss, second only to the conversion to BPD. Tran et al. in their review of 866 patients, have reported a $54 \%$ mean EWL one-year post-op and a respective rate of $52.2 \% 3$-years after the revision with a complication rate (mainly malnutrition) of $11.9 \%$ (29). The author's much smaller anecdotal experience with both types of revision is similar with an over $50 \%$ EWL and only one case of nutritional deficiency that had to be revised after an open type I conversion to DRYGBP.

Gastric Pouch-gastrojejunostomy Revision. Weight regain after RYGBP quite often is attributed to a defective function of the gastric pouch-gastro-enterostomy complex of the index operation. A gastro-gastric fistula was an infrequent finding after RYGBP before the perfection of surgical staplers and mainly in the era where the gastric pouch was not constructed with total division of the stomach and only stapling of the stomach was performed. Nowadays a gastrogastric fistula can rarely be seen and usually is the result of the spontaneous healing of a gastro-enterostomy leak early post-operatively or the imperfect division of the gastric fundus during the pouch construction (32).

Since the opening of a gastro-gastric fistula permits the passage of food not only through the gastro-jejunostomy (GJ) but also through the fistula towards the gastric remnant, this event totally negates both the mechanically restrictive consequences of the operation but also nullifies its' hormonal changes, since it allows the propagation of foods through the pylorus into the duodenum, thus totally destroying the inherent mechanisms of RYGBP for lowering the body weight.

Quite often, especially when there is a co-existent anastomotic stricture, the fistulous tract is wider than the gastro-enterostomy and more food passes through the fistula than through the anastomosis. In cases like this, correction of the obvious underlying problem is necessary, and this would usually mean resection of the apex of the pouch containing the fistula, normally along with the anastomosed intestinal loop and the construction of a new GJ.

Besides, much more frequently a dilation of the gastric pouch can be found in patients with inadequate weight loss or weight regain after RYGBP. This can either be the result of poor surgical technique during the initial operation or the result of bad eating habits, putting the gastric pouch under constant pressure that gradually dilates it, thus reducing the restrictive effect of the operation. The problem is that the definition of what is a clinically significant pouch enlargement is not well defined as of today. There are suggestions that a pouch longer than $6 \mathrm{~cm}$ or wider than 5 $\mathrm{cm}$ is dilated and the diameter of the GJ has to be kept under $1.5 \mathrm{~cm}$ (33), however, the association of these data with weight regain is rather vague (34).

Whenever a dilated gastric pouch is seen in an upper GI series in a patient with a failed gastric bypass, a revision aiming at the reduction of its size, thus re-establishing a drastic restriction in food intake seems like a promising concept. Various types of revisions have been described (Figure 3). A lateral resection of the gastric pouch only, a lateral resection of the pouch and part of the GJ, a resection of a dilated blind intestinal loop producing the so called "candy cane syndrome" or finally the resection of the whole GJ along with the dilated part of the pouch and the construction of a new GJ; all are options that have been described in the literature (35-37).

The initial weight loss after these types of revision has been reported to be good enough with an excess BMI loss of $43.1 \%$ after 12 months $(38,39)$. It seems though that this good initial result fades away in the mid-term, since 3-years post-op the excess BMI loss drops drastically to $14 \%$ or even a BMI increase has been reported $(40,41)$. 
Although the concept of resizing a dilated pouch looks promising, it resembles conceptually to re-positioning a failed band or even more to re-sleeving a dilated gastric sleeve. All these concepts originate in the assumption that by restoring a failed technical factor one can achieve the intended result. However, it can very well be that weight regain is not simply a matter of technical failure of the operation but of its' inappropriateness per se or that the poor compliance from the patient's part was responsible for the failure, and by trying to fix the erroneously identified technical factor as responsible for the suboptimal results, we simply repeat the same error for a second time. Our small anecdotal experience had ambiguous results with two midterm failures and one patient with $>50 \%$ excess weight loss more than 3 years post revision.

After all, revising the GJ laparoscopically can be technically challenging but usually is a more straight-forward laparoscopic operation than other surgical options for the treatment of weight regain after RYGBP and can be achieved with a more than acceptable $3.4 \%$ rate of post-op morbidity and no mortality.

Banding of the Gastric Pouch. Gastric banding as an initial procedure for the treatment of morbid obesity is a wellknown option with a popularity that has fluctuated from being the commonest bariatric procedure worldwide in the early 2000 's to a frequency of less than $10 \%$ today (8). The reasons for this discrepancy must be the trade-off between a good early safety record and a rather infamous long-term overall record, comprising suboptimal weight loss and minor or major complications necessitating revisional procedures for more than $50 \%$ of patients (42).

A banded variation of RYGBP, the so-called "Fobi" procedure, has also been described early on, as a possible alternative to standard RYGBP, in order to retain the good initial results concerning weight loss in the long run (43). This having be said, it seems reasonable that banding the gastric pouch can be suggested as a probable alternative for patients with weight regain after RYGBP (Figure 4).

The rationale is that by adding a band around the gastric pouch one can prevent its' post-prandial dilation, thus retaining the restrictive effect of the procedure. Indeed, data supporting this notion are available. Both the regular adjustable or a fixed type of band have been used with good early and mid-term results. Irani et al. have reported a $51 \%$ excess BMI loss after positioning a regular adjustable gastric band around the gastric pouch (44), while Dapri et al. have reported even a $70 \%$ EWL with a fixed silicone ring placed loosely around the pouch, thus reducing its' postprandial compliance (45).

The enthusiasts of the procedure claim universally that the overall complication rate of the technique is negligible, and this is its' main advantage. Regarding its' comparative

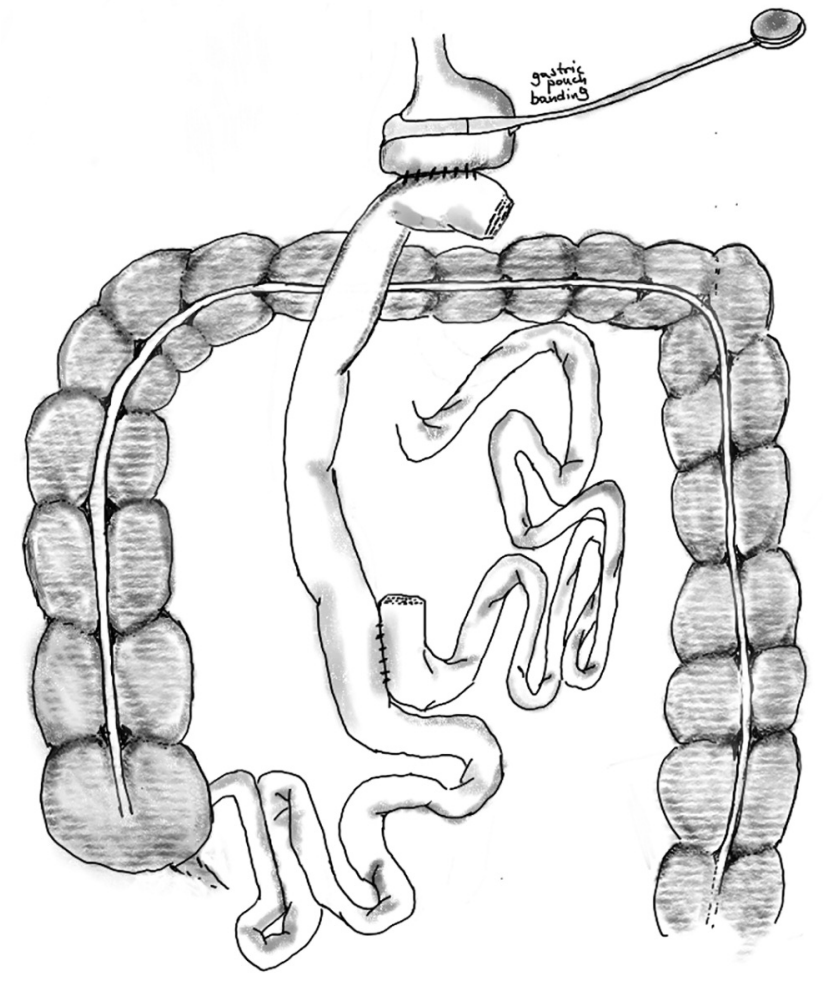

Figure 4. Gastric pouch banding.

efficacy concerning long-term weight loss, the conversion of standard to banded RYGBP ranks third behind the conversions to BPD-DS and DRYGBP and above the revision of the gastric pouch in the review of Tran et al. (29).

However, these results have to be met with some skepticism. Firstly, since the problems with the lap-band as a stand-alone procedure have been shown to be delayed, longer-term results must be awaited before this type of revision can be widely suggested. Recently, Liu et al. reporting on the long-term results of the Irani et al. series of patients reported a good $65 \%$ EWL five years after the conversion of standard to banded RYGBP but with a remarkable $24 \%$ reoperation and $8 \%$ removal rate (46). Similar results have been published by Dillemans et al. (47). Secondly, RYGBP has always been considered as one of the most elaborate, complicated, mature, and successful techniques for the treatment of morbid obesity, while lapband as a simple and rather primitive concept lagging clearly behind RYGBP in terms of long-term excess weight loss.

It seems rather unsound theoretically to add the lesser treatment when the greater has already failed in addressing what obviously is a resistant to treatment disease, therefore at least some caution about the long-term durability of what seems like good initial results is reasonable. 
Endoscopic Techniques. With the advent of new technologies, invasive endoscopists can today try to reproduce the results of surgery with intraluminal simulations of well-established surgical techniques. An obvious example of this tendency is POEM for the treatment of achalasia. Predictably, revisional procedures for weight regain after gastric bypass constitute predictably a prime target for invasive gastroenterologists.

Available options include transoral outlet reduction (TORe), restorative obesity surgery endoscopic (ROSE), endoscopic sclerotherapy, and endoscopic gastric plication (EGP). TORe is essentially the reduction of the diameter of the gastro-jejunal anastomosis with the placement of intraluminal sutures, which are then tightened with the use of the Overstitch Endoscopic Suturing System. ROSE is a quite similar technique involving the reduction of the size of the gastric pouch and the anastomotic surface with the use of H-shaped anchors creating tissue folds. Endoscopic sclerotherapy involves the injection of sclerosing agents in the anastomotic margins to create scars, thus inducing stenosis of a dilated GJ. Finally, endoscopic plication is a variation of the ROSE technique with the use of the Stomaphyx device.

All these techniques resemble each other and originate in the theory that a dilated GJ or an enlarged gastric pouch can be held responsible for the weight regain, therefore an effort towards a minimally invasive means of reconstituting a properly sized anatomy of the gastric pouch and the GJ suffices for the reversal of weight recidivism. In this way, they resemble surgical techniques such as revision or even banding of the gastric pouch. With the use of the TORe technique, a loss of $10.8 \mathrm{kgs}$ or $56.3 \%$ of the regained weight 1 -year post-endoscopy has been reported (48). However, in that paper no data concerning EWL, EBMIL or actual BMI were presented, thus a meaningful evaluation of the efficacy of the technique or comparison with the available alternatives was impossible.

Various reports of the ROSE technique have reported a rather disappointing 3 units of BMI lowering or a well below $50 \%$ EWL loss at 1-year post-intervention, thus casting doubts about the efficacy of the technique for the time being $(15,49)$. Endoscopic sclerosing of the GJ is technically simpler and less demanding, thus it may be more widely used, however its results concerning weight loss are less impressive. Even its supporters claim primarily the stabilization of the body weight rather than its reduction (50). The combination of endoscopic sclerotherapy with simultaneous endoluminal suturing has been reported to produce better mid-term results, especially when a GJ diameter less than $1 \mathrm{~cm}$ is achieved (51).

Finally, EGP has currently very mediocre or even negligible efficacy concerning regained excess weight. In a series of 27 patients, all of them weighed one year after the EGP exactly as much as they weighed before the revision and upon repeat endoscopy the vast majority of the folds created during the EGP were undone, suggesting that the technique as of today has not been perfected in order to be durable (52). Another problem with all these techniques is that as of today the studies reporting the relevant data are mainly feasibility studies, therefore the authors reporting the data focus on technical success, peri-operative morbidity, and weight loss in $\mathrm{kgs}$ and not in the common for the bariatric literature form of \%EWL or \%EBMIL (excess BMI loss), thus making impossible the comparative evaluation of their results.

Finally, since for the time being insurance coverage for these techniques has not been approved, the compensation for these endoscopic techniques poses a heavy financial burden on the patients. All these reasons concur to the conclusion that currently the endoscopic ways of treatment of weight regain after gastric bypass are under development and do not represent yet a reasonable alternative for the average patient or bariatric service.

Conversion to BPD-DS. Biliopancreatic diversion-duodenal switch (or even its European analogue biliopancreatic bypass, the so-called Scopinaro procedure) represents the most powerful tool in the armamentarium of bariatric surgeons to treat morbid or super-morbid obesity and ameliorate the associated co-morbidities (53). Its widespread use has been hampered by its complexity, an associated long learning curve involving increased morbidity and mortality, and a high incidence of long-term nutritional problems due to excessive malabsorption, necessitating frequent hospitalizations and revisional surgery to be alleviated (54).

For patients who have already failed RYGBP in terms of weight loss, at least in theory, it seems quite rational to be upgraded to such a procedure. The problem is that if BPDDS is a difficult initial procedure to master, it can be a huge challenge to be performed as a revisional procedure. In essence, it consists of two complementary, complex re-do operations. It involves the re-establishment of gastric continuity with the taking down of the GJ and the construction of a gastro-gastric anastomosis, a consecutive sleeve gastrectomy, the division of duodenum between its' first and second portion (Figure 5), the taking down of the $\mathrm{JJ}$ and the reconstitution of the intestinal continuity, and finally the fashioning of the duodeno-ileal anastomosis and the construction of a new JJ after having measured the length of terminal ileum in order to establish a 1-1.5 meter common channel with the creation of a long biliopancreatic loop (Figure 6).

The operation has been described both as a single-step and as a two-stage procedure. It is an obviously challenging surgical task, thus it is performed in very few centers and the reported number of patients are rather small. The group of Gagner has reported on 12 patients with this type of conversion back in 2007 (55). Similar results have been 

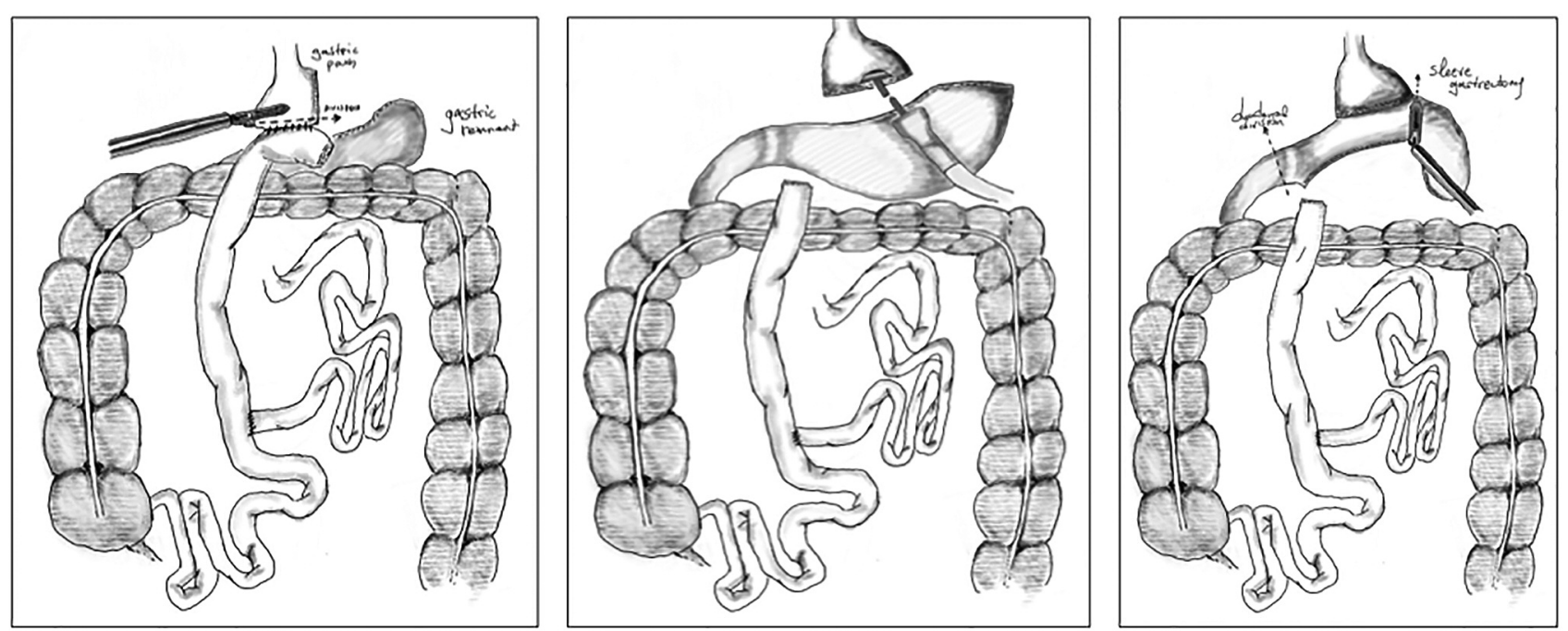

Figure 5. Division of the gastro-jejunostomy, gastrogastric anastomosis, sleeve gastrectomy.
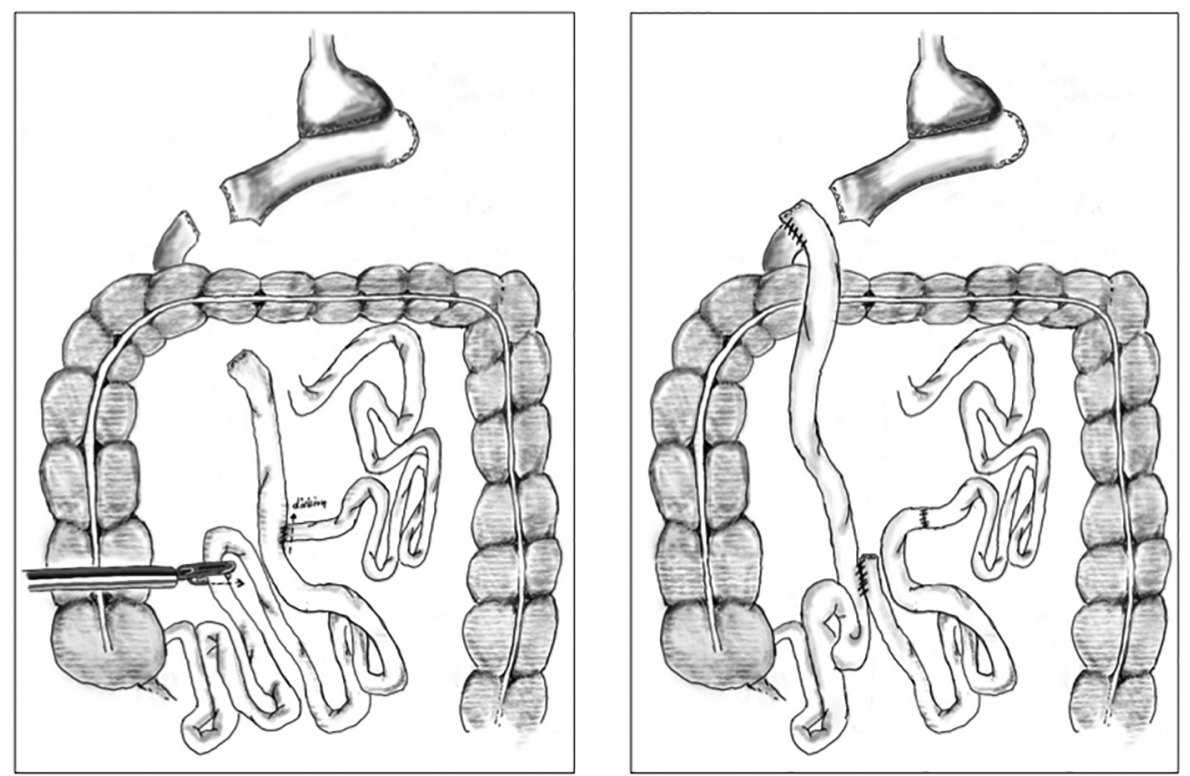

Figure 6. Conversion of Roux-en-Y gastric bypass to biliopancreatic diversion.

reported even earlier by Keshishian et al. in their review of the use of BPD-DS as a revisional procedure for weight loss failure after any type of bariatric surgery and the reported morbidity and mortality rates were $<5 \%$ and $1 \%$, respectively (56). Of course, these results have come from dedicated high-volume centers and are hardly reproducible in the less than highly specialized setting. Long-term results concerning excess weight loss are excellent. Nutritional deficiencies represent a real problem and necessitate a strict follow-up protocol.
Although this type of conversion probably represents the most reliable means of treatment for recurrent morbid obesity after RYGBP, the profound technical difficulties and its' associated early and long-term morbidity currently prevent its' widespread use outside the setting of highly specialized high-volume centers of excellence in bariatric surgery.

Single Anastomosis Operations. Single anastomosis variations of traditional bariatric techniques such as one anastomosis gastric bypass (OAGB) and single anastomosis 
duodeno-ileal bypass (SADI-s) have been advocated as contemporary safe and efficacious options of treatment for morbid obesity and have rapidly gained remarkable popularity considering their lack of long-term data or complexity. Their use as revisional procedures after RYGBP for inadequate weight loss has been sparsely reported, more as a technical exercise between the ranks of case reports or video presentation in conferences and less as a suggested high-scale treatment option (57). For the time being, there are no solid data allowing their comparison with the other already mentioned techniques.

\section{Conclusion}

The available data suggest that endoscopic procedures currently fail to produce significant short- and long-term weight loss for patients with weight regain after gastric bypass and only exceptionally can be offered in dedicated centers for dedicated patients, partly in an effort to improve the available techniques. Banding the gastric pouch seems like a simple technique with promising initial results. Disappointing long-term results of the lap-band as a standalone procedure make it imperative to await longer term results before widely advocating this technique. Reconstruction of the gastric pouch and the GJ have produced satisfactory early but less than so long-term results, thus although conceptually it looks like a very straight forward solution, its efficacy remains not well documented and must be used with caution. Conversion of RYGBP to BPD-DS produces the best results with acceptable peri-operative morbidity. The profound complexity of the procedure has prevented its widespread use, thus the existing data are rather few to draw definitive conclusions, while also deem it improbable that this operation will be offered in a large scale. Eventually, conversion of standard to distal gastric bypass by repositioning the $\mathrm{JJ}$ thus adding malabsorption, has been proven to be safe and efficient. The reposition of the JJ in a way that retains the length of biliopancreatic loop and only changes the ratio of alimentary to common channel length seems to carry a better profile concerning long-term nutritional status without significantly compromising the rate of weight loss, thus represents as of today the safest, effective solution for the average bariatric surgeon who faces an obese patient with weight regain after RYGBP.

\section{Conflicts of Interest}

All Authors declare no potential conflicts of interest in relation to this study.

\section{Authors' Contributions}

Study conception and design: Andreas Alexandrou, Spyridon Davakis and Evangelos Felekouras. Acquisition of data and analysis and interpretation of data: Andreas Alexandrou, Panagiotis Sakarellos, Spyridon Davakis, Michail Vailas, Nikoletta Dimitriou, Alexandros Papalampros, Dimitrios Schizas, Alexandros Charalabopoulos and Evangelos Felekouras. Drafting of Manusctript: Andreas Alexandrou, Panagiotis Sakarellos, Spyridon Davakis, Michail Vailas, Nikoletta Dimitriou, Alexandros Papalampros, Dimitrios Schizas, Alexandros Charalabopoulos and Evangelos Felekouras. Critical revision: Andreas Alexandrou, Panagiotis Sakarellos and Spyridon Davakis. All Authors approved the final version of the article.

\section{References}

1 Phillips BT and Shikora SA: The history of metabolic and bariatric surgery: Development of standards for patient safety and efficacy. Metabolism 79: 97-107, 2018. PMID: 29307519. DOI: 10.1016/j.metabol.2017.12.010

2 Böckelman C, Hahl T and Victorzon M: Mortality following bariatric surgery compared to other common operations in Finland during a 5-year period (2009-2013). A Nationwide Registry Study. Obes Surg 27(9): 2444-2451, 2017. PMID: 28382506. DOI: 10.1007/s11695-017-2664-Z

3 Duvoisin C, Favre L, Allemann P, Fournier P, Demartines N and Suter M: Roux-en-Y gastric bypass: Ten-year results in a cohort of 658 patients. Ann Surg 268(6): 1019-1025, 2018. PMID: 29194086. DOI: 10.1097/SLA.0000000000002538

4 Carlin AM, Zeni TM, English WJ, Hawasli AA, Genaw JA, Krause KR, Schram JL, Kole KL, Finks JF, Birkmeyer JD, Share $\mathrm{D}$, Birkmeyer NJ and Michigan Bariatric Surgery Collaborative: The comparative effectiveness of sleeve gastrectomy, gastric bypass, and adjustable gastric banding procedures for the treatment of morbid obesity. Ann Surg 257(5): 791-797, 2013. PMID: 23470577. DOI: 10.1097/SLA.0b013e3182879ded

5 Patel K, Askari A, Mamidanna R, Jain V and Adil T: Long-term BAROS scores and independent obesity-related co-morbidity predictors of failure after laparoscopic Roux-en-Y gastric bypass. Surg Obes Relat Dis 16(12): 1954-1960, 2020. PMID: 32958371. DOI: 10.1016/j.soard.2020.07.037

6 Mason EE and Ito C: Gastric bypass in obesity. Surg Clin North Am 47(6): 1345-1351, 1967. PMID: 6073761. DOI: 10.1016/ s0039-6109(16)38384-0

7 Angrisani L, Santonicola A, Iovino P, Formisano G, Buchwald $\mathrm{H}$ and Scopinaro N: Bariatric Surgery Worldwide 2013. Obes Surg 25(10): 1822-1832, 2015. PMID: 25835983. DOI: 10.1007/ s11695-015-1657-z

8 Angrisani L, Santonicola A, Iovino P, Vitiello A, Zundel N, Buchwald $\mathrm{H}$ and Scopinaro $\mathrm{N}$ : Bariatric surgery and endoluminal procedures: IFSO Worldwide Survey 2014. Obes Surg 27(9): 2279-2289, 2017. PMID: 28405878. DOI: 10.1007/ s11695-017-2666-x

9 dos Santos Moraes I Jr, Madalosso CA, Palma LA, da Silva Fornari AC, do Socorro Dourado M, Scherer T, Gurski RR and Fornari F: Hospital discharge in the day following open Roux-enY gastric bypass: is it feasible and safe? Obes Surg 19(3): 281286, 2009. PMID: 19067087. DOI: 10.1007/s11695-008-9779-1

10 Sugerman HJ, Wolfe LG, Sica DA and Clore JN: Diabetes and hypertension in severe obesity and effects of gastric bypassinduced weight loss. Ann Surg 237(6): 751-6; discussion 757-8, 2003. PMID: 12796570. DOI: 10.1097/01.SLA.0000071560. 76194.11 
11 Longitudinal Assessment of Bariatric Surgery (LABS) Consortium., Flum DR, Belle SH, King WC, Wahed AS, Berk P, Chapman W, Pories W, Courcoulas A, McCloskey C, Mitchell J, Patterson E, Pomp A, Staten MA, Yanovski SZ, Thirlby R and Wolfe B: Perioperative safety in the longitudinal assessment of bariatric surgery. N Engl J Med 361(5): 445-454, 2009. PMID: 19641201. DOI: $10.1056 /$ NEJMoa0901836

12 Buchwald $\mathrm{H}$ and Buchwald $\mathrm{JN}$ : Metabolic (bariatric and nonbariatric) surgery for Type 2 diabetes: A personal perspective review. Diabetes Care 42(2): 331-340, 2019. PMID: 30665965. DOI: $10.2337 / \mathrm{dc} 17-2654$

13 Lim CS, Liew V, Talbot ML, Jorgensen JO and Loi KW: Revisional bariatric surgery. Obes Surg 19(7): 827-832, 2009. PMID: 18972173. DOI: 10.1007/s11695-008-9750-1

14 Kalarchian MA, Marcus MD, Wilson GT, Labouvie EW, Brolin RE and LaMarca LB: Binge eating among gastric bypass patients at long-term follow-up. Obes Surg 12(2): 270275, 2002. PMID: 11975227. DOI: 10.1381/ 096089202762552494

15 Horgan S, Jacobsen G, Weiss GD, Oldham JS Jr, Denk PM, Borao F, Gorcey S, Watkins B, Mobley J, Thompson K, Spivack A, Voellinger D, Thompson C, Swanstrom L, Shah P, Haber G, Brengman $M$ and Schroder G: Incisionless revision of postRoux-en-Y bypass stomal and pouch dilation: multicenter registry results. Surg Obes Relat Dis 6(3): 290-295, 2010. PMID: 20510293. DOI: 10.1016/j.soard.2009.12.011

16 Arman GA, Himpens J, Dhaenens J, Ballet T, Vilallonga R and Leman G: Long-term (11+years) outcomes in weight, patient satisfaction, comorbidities, and gastroesophageal reflux treatment after laparoscopic sleeve gastrectomy. Surg Obes Relat Dis 12(10): 1778-1786, 2016. PMID: 27178613. DOI: 10.1016/ j.soard 2016.01 .013

17 Brolin RE: Bariatric surgery and long-term control of morbid obesity. JAMA 288(22): 2793-2796, 2002. PMID: 12472304 DOI: $10.1001 /$ jama.288.22.2793

18 Kellum JM, Chikunguwo SM, Maher JW, Wolfe LG and Sugerman HJ: Long-term results of malabsorptive distal Rouxen-Y gastric bypass in superobese patients. Surg Obes Relat Dis 7(2): 189-193, 2011. PMID: 21145293. DOI: 10.1016/j.soard. 2010.08.018

19 Fobi MA, Lee H, Igwe D Jr, Felahy B, James E, Stanczyk M, Tambi J and Eyong P: Revision of failed gastric bypass to distal Roux-en-Y gastric bypass: a review of 65 cases. Obes Surg 11(2): 190-195, 2001. PMID: 11355025. DOI: 10.1381/ 096089201321577866

20 Rawlins ML, Teel D 2nd, Hedgcorth K and Maguire JP: Revision of Roux-en-Y gastric bypass to distal bypass for failed weight loss. Surg Obes Relat Dis 7(1): 45-49, 2011. PMID: 21111688. DOI: $10.1016 /$ j.soard .2010 .08 .013

21 Srikanth MS, Oh KH and Fox SR: Revision to malabsorptive Roux-en-Y gastric bypass (MRNYGBP) provides long-term (10 years) durable weight loss in patients with failed anatomically intact gastric restrictive operations: long-term effectiveness of a malabsorptive Roux-en-Y gastric bypass in salvaging patients with poor weight loss or complications following gastroplasty and adjustable gastric bands. Obes Surg 21(7): 825-831, 2011. PMID: 20835895. DOI: 10.1007/s11695-010-0280-2

22 Fox SR, Fox KS and Oh KH: The gastric bypass for failed bariatric surgical procedures. Obes Surg 6(2): 145-150, 1996. PMID: 10729855. DOI: 10.1381/096089296765557097
23 Brolin RE and Cody RP: Weight loss outcome of revisional bariatric operations varies according to the primary procedure. Ann Surg 248(2): 227-232, 2008. PMID: 18650632. DOI: 10.1097/SLA.0b013e3181820cdf

24 Torres JC: Why i prefer gastric bypass distal Roux-en-Y gastroileostomy. Obes Surg 1(2): 189-194, 1991. PMID: 10775917. DOI: $10.1381 / 096089291765561268$

25 Sugerman HJ, Kellum JM and DeMaria EJ: Conversion of proximal to distal gastric bypass for failed gastric bypass for superobesity. J Gastrointest Surg 1(6): 517-24; discussion 524-6, 1997. PMID: 9834387. DOI: 10.1016/s1091-255x(97)80067-4

26 Brolin RE and Cody RP: Adding malabsorption for weight loss failure after gastric bypass. Surg Endosc 21(11): 1924-1926, 2007. PMID: 17768659. DOI: 10.1007/s00464-007-9542-z

27 Dapri G, Cadière GB and Himpens J: Laparoscopic conversion of Roux-en-Y gastric bypass to distal gastric bypass for weight regain. J Laparoendosc Adv Surg Tech A 21(1): 19-23, 2011. PMID: 21138345. DOI: 10.1089/lap.2010.0298

28 Buchwald H and Oien DM: Revision Roux-en-Y gastric bypass to biliopancreatic long-limb gastric bypass for inadequate weight response: case series and analysis. Obes Surg 27(9): 2293-2302, 2017. PMID: 28405877. DOI: 10.1007/s11695-017-2658-x

29 Tran DD, Nwokeabia ID, Purnell S, Zafar SN, Ortega G, Hughes $\mathrm{K}$ and Fullum TM: Revision of Roux-En-Y gastric bypass for weight regain: a systematic review of techniques and outcomes. Obes Surg 26(7): 1627-1634, 2016. PMID: 27138603. DOI: 10.1007/s11695-016-2201-5

30 Kraljević M, Köstler T, Süsstrunk J, Lazaridis II, Taheri A, Zingg U and Delko T: Revisional surgery for insufficient loss or regain of weight after Roux-en-Y gastric bypass: Biliopancreatic limb length matters. Obes Surg 30(3): 804-811, 2020. PMID: 31863410. DOI: $10.1007 / \mathrm{s} 11695-019-04348-8$

31 Shin RD, Goldberg MB, Shafran AS, Shikora SA, Majumdar MC and Shikora SA: Revision of Roux-en-Y gastric bypass with limb distalization for inadequate weight loss or weight regain. Obes Surg 29(3): 811-818, 2019. PMID: 30560312. DOI: $10.1007 / \mathrm{s} 11695-018-03635-0$

32 Carrodeguas L, Szomstein S, Soto F, Whipple O, Simpfendorfer C, Gonzalvo JP, Villares A, Zundel $\mathrm{N}$ and Rosenthal R: Management of gastrogastric fistulas after divided Roux-en-Y gastric bypass surgery for morbid obesity: analysis of 1,292 consecutive patients and review of literature. Surg Obes Relat Dis 1(5): 467-474, 2005. PMID: 16925272. DOI: 10.1016/ j.soard.2005.07.003

33 Brethauer SA, Nfonsam V, Sherman V, Udomsawaengsup S, Schauer PR and Chand B: Endoscopy and upper gastrointestinal contrast studies are complementary in evaluation of weight regain after bariatric surgery. Surg Obes Relat Dis 2(6): 643-8; discussion 649-50, 2006. PMID: 17020823. DOI: 10.1016/ j.soard.2006.07.012

34 Heneghan HM, Yimcharoen P, Brethauer SA, Kroh M and Chand B: Influence of pouch and stoma size on weight loss after gastric bypass. Surg Obes Relat Dis 8(4): 408-415, 2012. PMID: 22055390. DOI: 10.1016/j.soard.2011.09.010

35 Borbély Y, Winkler C, Kröll D and Nett P: Pouch reshaping for significant weight regain after Roux-en-Y gastric bypass. Obes Surg 27(2): 439-444, 2017. PMID: 27510586. DOI: 10.1007/ s11695-016-2329-3

36 Elbahrawy A, Bougie A, Albader M, Aggarwal R, Demyttenaere $\mathrm{S}$, Andalib A and Court O: Laparoscopic wedge resection of 
gastrojejunostomy for weight recidivism after gastric bypass Obes Surg 27(11): 2829-2835, 2017. PMID: 28470487. DOI: 10.1007/s11695-017-2706-6

37 Dallal RM and Cottam D: "Candy cane" Roux syndrome-a possible complication after gastric bypass surgery. Surg Obes Relat Dis 3(3): 408-410, 2007. PMID: 17533103. DOI: 10.1016/ j.soard.2007.02.011

38 Morales MP, Wheeler AA, Ramaswamy A, Scott JS and de la Torre RA: Laparoscopic revisional surgery after Roux-en-Y gastric bypass and sleeve gastrectomy. Surg Obes Relat Dis 6(5): 485-490, 2010. PMID: 20870181. DOI: 10.1016/j.soard. 2009.09.022

39 Iannelli A, Schneck AS, Hébuterne X and Gugenheim J: Gastric pouch resizing for Roux-en-Y gastric bypass failure in patients with a dilated pouch. Surg Obes Relat Dis 9(2): 260-267, 2013. PMID: 22695174. DOI: 10.1016/j.soard.2012.05.003

40 Parikh M, Heacock L and Gagner M: Laparoscopic "gastrojejunal sleeve reduction" as a revision procedure for weight loss failure after roux-en-y gastric bypass. Obes Surg 21(5): 650-654, 2011. PMID: 20835779. DOI: 10.1007/s11695010-0274-0

41 Hamdi A, Julien C, Brown P, Woods I, Hamdi A, Ortega G, Fullum $\mathrm{T}$ and Tran D: Midterm outcomes of revisional surgery for gastric pouch and gastrojejunal anastomotic enlargement in patients with weight regain after gastric bypass for morbid obesity. Obes Surg 24(8): 1386-1390, 2014. PMID: 24634099. DOI: $10.1007 / \mathrm{s} 11695-014-1216-\mathrm{z}$

42 Himpens J, Cadière GB, Bazi M, Vouche M, Cadière B and Dapri G: Long-term outcomes of laparoscopic adjustable gastric banding. Arch Surg 146(7): 802-807, 2011. PMID: 21422330. DOI: $10.1001 /$ archsurg. 2011.45

43 Fobi MA, Lee H, Felahy B, Che K, Ako P and Fobi N: Choosing an operation for weight control, and the transected banded gastric bypass. Obes Surg 15(1): 114-121, 2005. PMID: 15760509. DOI: 10.1381/0960892052993404

44 Irani K, Youn HA, Ren-Fielding CJ, Fielding GA and Kurian M: Midterm results for gastric banding as salvage procedure for patients with weight loss failure after Roux-en-Y gastric bypass. Surg Obes Relat Dis 7(2): 219-224, 2011. PMID: 21130044. DOI: $10.1016 /$ j.soard .2010 .09 .024

45 Dapri G, Cadière GB and Himpens J: Laparoscopic placement of non-adjustable silicone ring for weight regain after Roux-enY gastric bypass. Obes Surg 19(5): 650-654, 2009. PMID: 19263180. DOI: $10.1007 / \mathrm{s} 11695-009-9807-9$

46 Liu S, Ren-Fielding CJ, Schwack B, Kurian M and Fielding GA: Long-term results for gastric banding as salvage procedure for patients with weight loss failure after Roux-en-Y gastric bypass. Surg Obes Relat Dis 14(10): 1501-1506, 2018. PMID: 30154032. DOI: 10.1016/j.soard.2018.07.019

47 Jacobs K, Vleeschouwers W, Debergh I, Haesen D and Dillemans B: Laparoscopic adjustable gastric banding with the Adhesix ${ }^{\circledR}$ Bioring ${ }^{\circledR}$ for weight regain or insufficient weight loss after a Roux-en-Y gastric bypass: Midterm data from the pronto registry. Obes Surg 31(10): 4295-4304, 2021. PMID: 34275109. DOI: $10.1007 / \mathrm{s} 11695-021-05537-0$
48 Jirapinyo P, Slattery J, Ryan MB, Abu Dayyeh BK, Lautz DB and Thompson CC: Evaluation of an endoscopic suturing device for transoral outlet reduction in patients with weight regain following Roux-en-Y gastric bypass. Endoscopy 45(7): 532-536, 2013. PMID: 23801313. DOI: 10.1055/s-0032-1326638

49 Ryou M, Mullady DK, Lautz DB and Thompson CC: Pilot study evaluating technical feasibility and early outcomes of secondgeneration endosurgical platform for treatment of weight regain after gastric bypass surgery. Surg Obes Relat Dis 5(4): 450-454, 2009. PMID: 19632645. DOI: 10.1016/j.soard.2009.03.217

50 Abu Dayyeh BK, Jirapinyo P, Weitzner Z, Barker C, Flicker MS, Lautz DB and Thompson CC: Endoscopic sclerotherapy for the treatment of weight regain after Roux-en-Y gastric bypass: outcomes, complications, and predictors of response in 575 procedures. Gastrointest Endosc 76(2): 275-282, 2012. PMID: 22817783. DOI: $10.1016 /$ j.gie.2012.03.1407

51 Riva P, Perretta $S$ and Swanstrom L: Weight regain following RYGB can be effectively treated using a combination of endoscopic suturing and sclerotherapy. Surg Endosc 31(4): 1891-1895, 2017. PMID: 27553793. DOI: 10.1007/s00464-0165189-y

52 Ong'uti SK, Ortega G, Onwugbufor MT, Ivey GD, Fullum TM and Tran DD: Effective weight loss management with endoscopic gastric plication using StomaphyX device: is it achievable? Surg Obes Relat Dis 9(1): 113-117, 2013. PMID: 22169759. DOI: 10.1016/j.soard.2011.08.025

53 Skogar ML and Sundbom M: Duodenal switch is superior to gastric bypass in patients with super obesity when evaluated with the bariatric analysis and reporting outcome system (BAROS). Obes Surg 27(9): 2308-2316, 2017. PMID: 28439748. DOI: $10.1007 / \mathrm{s} 11695-017-2680-\mathrm{Z}$

54 Anderson B, Gill RS, de Gara CJ, Karmali S and Gagner M: Biliopancreatic diversion: the effectiveness of duodenal switch and its limitations. Gastroenterol Res Pract 2013: 974762, 2013. PMID: 24639868. DOI: 10.1155/2013/974762

55 Parikh M, Pomp A and Gagner M: Laparoscopic conversion of failed gastric bypass to duodenal switch: technical considerations and preliminary outcomes. Surg Obes Relat Dis 3(6): 611-618, 2007. PMID: 17936087. DOI: 10.1016/j.soard.2007.07.010

56 Keshishian A, Zahriya K, Hartoonian $\mathrm{T}$ and Ayagian C: Duodenal switch is a safe operation for patients who have failed other bariatric operations. Obes Surg 14(9): 1187-1192, 2004. PMID: 15527632. DOI: 10.1381/0960892042387066

57 Antozzi L, Pérez-Santillán JA, Abreu Quezada H, Luque-deLeón E and Carbajo MÁ: Roux-en-Y to one anastomosis gastric bypass conversion: Description of a novel technique. J Laparoendosc Adv Surg Tech A, 2021. PMID: 34534011. DOI: 10.1089/lap.2021.0518 